\title{
Validity of oral mucosal transudate specimens for HIV testing using enzyme- linked immunosorbent assay in children in Chimanimani district, Zimbabwe
}

\author{
Wilson Mashange, Stella May Gwini, Stanford T Mahati, Stephen S Buzuzi, Chenjerai K Mutambanengwe, Shungu Munyati,
} Brian Chandiwana, Simbarashe Rusakaniko, Exnevia Gomo

Objective. To assess the validity of oral mucosal transudate (OMT) specimens for HIV testing in children using enzyme-linked immunosorbent assay (ELISA).

Methods. A cross-sectional descriptive study was conducted as part of a community-based behavioural and HIV sero-status survey of adults and children in the Chimanimani district of Zimbabwe. Dried blood spot (DBS) and OMT samples were collected from children aged between 2 and 14 years, inclusive. Both samples were tested for HIV using the Vironostika Uniform II plus O kits. The main study outcomes were the sensitivity and specificity of OMT samples, with DBS as the gold-standard specimen.

Results. Paired DBS and OMT specimens were available from $1274(94.4 \%)$ of the 1350 children enrolled. Using the DBS, HIV prevalence was $3.2 \%$. Overall sensitivity of OMT was $48.8 \%$ (95\% confidence interval (CI) 33.3 - 64.5), and specificity was $98.5 \%$ (95\% CI 97.7 - 99.1).

Conclusion. The overall sensitivity of OMT specimens for HIV testing in children using ELISA was low. Stratifying the analysis by sector showed that OMT samples are good specimens for HIV testing. It is important to note that factors such as the low HIV prevalence in our study population, quality of the OMT, diet and oral hygiene could have influenced the results.

S Afr Med J 2011; 101: 49-52.
Nearly $70 \%$ of all human immunodeficiency virus (HIV) infections are in sub-Saharan Africa, an area that is home to only $10 \%$ of the world's population. ${ }^{1}$ In Zimbabwe the prevalence of HIV peaked

Biomedical Research and Training Institute, Zimbabwe, and National Institute of Health Research, Ministry of Health and Child Welfare, Zimbabwe Wilson Mashange, Diploma Applied Biological Technology, Certificate Medical Laboratory Technology

Biomedical Research and Training Institute, Zimbabwe, and Monash University, Australia

Stella May Gwini, MSc, BSc Hons (Statistics)

Biomedical Research and Training Institute, Zimbabwe, and School of Social Sciences, University of the Witwatersrand, Johannesburg, South Africa

Stanford T Mahati, MPhil, BSc Hons (Sociology)

Biomedical Research and Training Institute, Zimbabwe

Stephen S Buzuzi, MBA, MSc, BSc Hons (Sociology)

Biomedical Research and Training Institute, Zimbabwe, and Hasselt University, Belgium

Chenjerai K Mutambanengwe, BSc Hons (Applied Mathematics), MSc Biostatistics

Biomedical Research and Training Institute and National Institute of Health Research, Ministry of Health and Child Welfare, Zimbabwe

Shungu Munyati, MSc, BSc Hons (Applied Biology)

Biomedical Research and Training Institute, Zimbabwe

Brian Chandiwana, MBA, BSc (Economics)

Department of Community Medicine, College of Health Sciences, University of Zimbabwe

Simbarashe Rusakaniko, PhD, MSc, BSc Hons (Statistics)

Research Support Centre, College of Medicine, University of Malawi, and Department of Immunology, College of Health Sciences, University of Zimbabwe

Exnevia Gomo, PhD, MSc (Applied Immunology) at $33 \%$ in $2001^{2}$ and had declined to $15.3 \%$ by $2007 .{ }^{1}$ Nevertheless, between 1.2 and 1.4 million people, including children, were living with HIV and AIDS in Zimbabwe in $2007 .{ }^{1}$

Most HIV prevalence data for children are inferred indirectly, because it is difficult to collect blood specimens from them. Alternative specimens with simpler and less invasive collection techniques are therefore needed to generate accurate data and improve access to antiretroviral therapy (ART) by children. While blood remains the most reliable specimen for HIV testing, oral mucosal transudate (OMT) specimens produce reliable results when collected for HIV testing using the Orasure device and tested with the enzyme-linked immunosorbent assay (ELISA). ${ }^{3}$ OMT is a salivary component from the capillaries of the gingival gum margin and has a high concentration of immunoglobulin G (IgG). Sensitivity and specificity of OMT specimens in HIV testing are high in adults, ${ }^{4-5}$ but it is inappropriate to infer these results to their use in children. Their validity in HIV diagnosis in infants has been assessed in South Africa, with sensitivities of $82 \%$ and $95 \% .{ }^{6-7}$ In contrast, a study in Uganda ${ }^{8}$ found low sensitivity for OMT specimens from children.

Collection of OMT specimens is non-invasive, thereby eliminating adverse effects associated with venepuncture (through both occupational exposure ${ }^{9}$ and accidental piercing by discarded needles ${ }^{10}$ ), is more acceptable to participants, ${ }^{11}$ and in some cases decreases costs ${ }^{12}$ since it can be used easily outside the clinic. In addition, collection and storage are simple. Factors influencing the accuracy and clinical utility of OMT as a specimen include the HIV prevalence in the study population, clinical conditions, and the type of population being tested. ${ }^{13}$ Other factors include quality control and quality assurance procedures during collection, and storage and testing of the samples. ART and low levels of seroconversion can result in false-negative results, and some medical conditions, bacterial contamination and elevated bilirubin can interfere with the test results. ${ }^{14}$

The value of OMT as a diagnostic specimen in HIV infection has not been assessed in Zimbabwe, and few studies have tested the validity of the specimen for HIV testing among children. Regardless of the HIV test method applied, OMT is likely to be advantageous since it is more acceptable to children than collection of blood. OMT samples were therefore collected from children to assess their 
sensitivity and specificity in HIV diagnosis using the Vironostika Uniform II commercial HIV-1/2 plus O kit.

\section{Methods}

This study was part of a 2004 cross-sectional behavioural risk and HIV sero-status survey conducted in Chimanimani district, Zimbabwe. Children between the ages of 2 and 14 years, inclusive, were enrolled. A structured questionnaire was administered by research assistants to parents/guardians of the 2 - 11-year-old children and directly to children aged $12-14$ years.

\section{Specimen collection and testing}

DBS specimens were collected on Whatman No.3 filter paper, following instructions from Whatman International. ${ }^{15}$ The blood spots were air-dried for at least 15 minutes away from direct sunlight and placed in appropriately labelled paper envelopes. OMT specimens were collected using the OraSure HIV-1 Oral Specimen Collection Device, following the manufacturer's instructions (Organon Technika, Netherlands). ${ }^{16}$ Before transportation to the laboratory, the DBS specimens were stored in a cool, dry place and the OMT specimens at room temperature. OMT specimens have been shown to be stable at temparatures below $37^{\circ} \mathrm{C}$ for at least 21 days. ${ }^{17}$ In the laboratory, all specimens were stored at $-20^{\circ} \mathrm{C}$.

All OMT specimens were tested within 6 weeks of collection. The Vironostika Uniform II plus O (BioMérieux, Netherlands) commercial ELISA kit, which is licensed for use with the Orasure collection device, ${ }^{18}$ was used to test for the presence of HIV antibodies. A similar Vironostika Uniform II kit routinely used with blood products was used to assay the DBS following the manufacturer's instructions.

\section{Quality control}

Quality and quantity of specimens were checked in the field and in the laboratory. All specimens were transferred to the laboratory within 14 days of collection. Quality checks included verification of labelling, quantity of each specimen, and checking for contamination of specimens.

Sufficiency of the DBS was determined by the size of the spot and whether the filter paper was soaked. A white filter-paper punch within 24 hours signified complete elution. Positive and negative control specimens provided by the manufacturer of the testing kits were used to assess intra-run and inter-run variation. In-house controls were also used, and Levy-Jennings charts were constructed (results not shown). Any analytical run (ELISA plate) that violated the standards was rejected, regardless of results obtained for other control materials. The controls were also sent to an external laboratory as part of a national programme of the Zimbabwe National Quality Assuarance Programme.

\section{Ethical considerations}

The study was approved by the Medical Research Council of Zimbabwe and by local and traditional leaders in Chimanimani. Participation in the study and collection of both the DBS and OMT specimens was voluntary. Written informed consent was sought from the children's parents/guardians and assent from the children. HIV testing was anonymous, although bar codes were used in order to link the questionnaire, DBS and OMT specimens. Parents/guardians who wanted to know the results of the tests were referred to the nearest district voluntary counselling and testing centre.

\section{Data analysis}

Data were entered using EPI Info version 6 and analysed using both SPSS 8.0 and STATA 7.0. Descriptive statistics were presented as medians (with lower and upper quartiles) for interval data and frequencies for categorical data. The chi-square test for association was used to check for associations between categorical variables. Evaluation of sensitivity, specificity, positive predictive value (PPV), negative predictive value (NPV) and test efficiency (TE) was done to determine the validity of the OMT specimen in HIV testing with DBS as the gold standard. TE refers to the overall ability of a test to correctly identify all positives and negatives, i.e. total effectiveness of an assay.

A detailed description of the methodology is provided in a report by Gomo et al. ${ }^{19}$

\section{Results}

\section{Characteristics of the study population}

A total of 1819 children were approached; 1350 (74.2\%) agreed to participate and provided DBS specimens. Of the 1350 DBS specimens collected, 1290 (95.6\%) were considered sufficient for HIV testing. OMT specimens were collected from 1335 (73.4\%) of the 1819 children who were approached. Of these, 1334 (99.9\%) were considered sufficient for HIV testing. A total of 1274 children provided specimens that were adequate as both DBS and OMT samples, and the remainder of the analysis presented refers only to these children (Fig. 1).

The median age of the 1274 children who had paired DBS and OMT specimens was 10 years (lower quartile 5, upper quartile 13), and $46.6 \%$ were aged $12-14$ years. There was an equal distribution of males and females (Table I), and $49.4 \%$ of children were from the communal areas and $24.6 \%$ from the resettlement areas.

\section{HIV prevalence}

Using the DBS as the gold standard test, HIV prevalence was 3.2\% overall. Prevalence was highest in the 6 - 8-year age group, but the difference according to age group was not statistically significant $(p=0.168)$. The prevalence was twice as high in females as in males (4.2 v. $2.2 \% ; p=0.040)$.

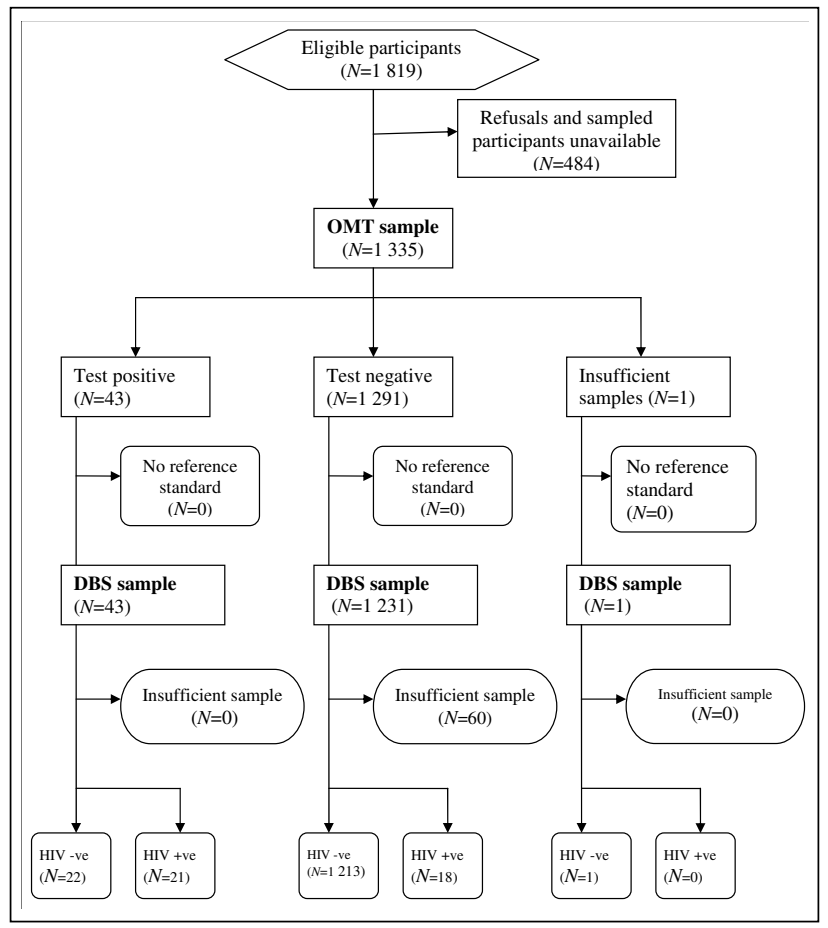

Fig. 1. Flow chart showing recruited participants. 


\begin{tabular}{|c|c|c|c|}
\hline & $N$ & $\begin{array}{l}\text { Males } \\
(N(\%))\end{array}$ & $\begin{array}{l}\text { Females } \\
(N(\%))\end{array}$ \\
\hline Total & 1274 & 638 & 636 \\
\hline \multicolumn{4}{|l|}{ Age group (yrs) } \\
\hline $2-5$ & $323(25.4)$ & $172(53.3)$ & $151(46.7)$ \\
\hline $6-8$ & $188(14.8)$ & $90(47.9)$ & $98(52.1)$ \\
\hline $9-11$ & $169(13.3)$ & $73(43.2)$ & $96(56.8)$ \\
\hline $12-14$ & $594(46.6)$ & $303(51.0)$ & $291(49.0)$ \\
\hline \multicolumn{4}{|l|}{ Sector } \\
\hline Communal & $629(49.4)$ & $312(49.6)$ & $317(50.4)$ \\
\hline Resettlement & $313(24.6)$ & $155(50.5)$ & $158(50.5)$ \\
\hline LSC & $152(11.9)$ & $72(47.4)$ & $80(52.6)$ \\
\hline SSC & $63(4.9)$ & $36(57.1)$ & $27(42.9)$ \\
\hline Urban & $117(9.2)$ & $63(53.9)$ & $54(46.1)$ \\
\hline
\end{tabular}

\section{Sensitivity and specificity of OMT}

According to OMT specimens, 43 children tested positive for HIV. Of the 43 , only 21 (48.8\%) also tested positive on DBS specimens. Of the 1231 children who tested HIV negative on OMT specimens, 18 were positive according to DBS results (Table II).

Overall sensitivity of OMT was therefore low (48.8\%), while specificity was high (98.5\%) (Table III). The test efficiency was $96.8 \%$ (95\% confidence interval (CI) 95.8 - 97.8). At a prevalence of 3.2\%, the positive predictive value (PPV) was $52.4 \%$ and the negative predictive value $98.3 \%$.

Sensitivity was low (less than 70\%) in most of the sectors, but it was higher than the overall for all the sectors except the urban sector (take note of the large CIs). Two wards (one rural, one urban) had much higher discordant results than other wards. Further analysis excluding the two wards gave a sensitivity of $70.4 \%$ (95\% CI 49.8 86.5), and specificity increased to $99.7 \%$ (95\% CI 99.2 - 99.9) and PPV to $89.0 \%$.

\section{Discussion}

Serum, plasma or dried blood spots are the most commonly used specimens for HIV diagnosis in Zimbabwe and the rest of the world. These specimens are difficult to collect from children, especially infants. OMT collection is less invasive than blood collection, may have fewer culturally linked misconceptions, and may therefore be more acceptable than DBS, serum and plasma. ${ }^{20}$ It could therefore increase the participation rate among children.

Sensitivity, specificity and other diagnostic parameters of OMT were assessed using DBS as the gold standard specimen. Overall sensitivity of OMT specimens was very low at $48.8 \%$, but specificity was high $(98.5 \%)$. While low sensitivity for children has also been observed in another study, ${ }^{8}$ sensitivities above $80 \%$ among infants were found in two studies. ${ }^{6,7}$ The prevalence of HIV infection in the latter was much higher (about 11\%) than that observed in this study (3.2\%), so the low HIV prevalence could have influenced the senstivity of the OMT specimens. ${ }^{13}$ The low sensitivity of OMT specimens in HIV testing in children in this study could have resulted from the children moving during specimen collection, so that saliva instead of OMT was collected, as suggested by Emmons et al..$^{10}$ Although IgG was not measured in this study, low levels of IgG in OMT could also have resulted in the low sensitivity. ${ }^{20,21}$ The positive predictive value of $52.4 \%$ indicates that under the conditions of this study, OMT specimens are not suitable for HIV screening in children.

Variations in the sensitivity of OMT specimens between this and other studies could also be due to differences in study settings. In most of the studies that have shown high sensitivities, specimens were from blood banks and public health, medical and STI/HIV clinics, whereas we collected our specimens in the households, outside clinical settings. Other factors contributing to the discrepancy could include dietary factors and oral hygiene. It is worth noting that the test efficiency, which refers to the total effectiveness of an assay, was high (96.8\%).

\section{Conclusion}

The overall sensitivity of OMT specimens for HIV testing in children using ELISA was low. Stratifying the analysis by sector showed that OMT may be a good specimen for HIV testing. The reasons for the sectoral and age differences could be technical or biological, and require further investigation. It is important to note that factors such as low HIV prevalence in our study population, quality of the OMT, diet and oral hygiene may have influenced the results.

We sincerely thank the participants and the local authority leaders, without whom this study could not have been successful. We also thank the W K Kellogg Foundation for funding the study, and the Human Sciences Research Council, South Africa, the Biomedical Research and Training Institute and the National Institute of Health Research of the Ministry of Health and Child Welfare, Zimbabwe, for the resources they provided. Special mention goes to Mrs J Mutsvangwa, Mrs J Magwenzi,

Table II. Concordance and discordance of the test results according to sector (N (\%))

\begin{tabular}{|c|c|c|c|c|c|c|}
\hline & \multicolumn{5}{|c|}{ Sector } & \multirow[b]{2}{*}{$\begin{array}{l}\text { Overall } \\
(N=1274)\end{array}$} \\
\hline & $\begin{array}{l}\text { Communal } \\
(N=629)\end{array}$ & $\begin{array}{l}\text { Resettlement } \\
(N=313)\end{array}$ & $\begin{array}{l}\text { LSC } \\
(N=152)\end{array}$ & $\begin{array}{l}\text { SSC } \\
(N=63)\end{array}$ & $\begin{array}{l}\text { Urban } \\
(N=117)\end{array}$ & \\
\hline DBS neg. + OMT neg. & $602(95.7)$ & 305 (97.4) & $145(95.4)$ & $62(98.4)$ & $98(83.8)$ & $1213(95.2)$ \\
\hline DBS pos. + OMT pos. & $9(1.4)$ & $5(1.6)$ & $4(2.6)$ & $1(1.6)$ & $2(1.7)$ & $21(1.7)$ \\
\hline DBS pos. + OMT neg. & $9(1.4)$ & $0(0.0)$ & $1(0.7)$ & $0(0.0)$ & $8(6.8)$ & $18(1.4)$ \\
\hline DBS neg. + OMT pos. & $9(1.4)$ & $3(1.0)$ & $2(1.3)$ & $0(0.0)$ & $9(7.7)$ & $22(1.7)$ \\
\hline
\end{tabular}


Table III. Sensitivity of OMT according to age group and sector

\begin{tabular}{|c|c|c|c|c|c|}
\hline & Sensitivity (\% (95\% CI)) & Specificity (\% (95\% CI)) & PPV (\%) & NPV (\%) & Test efficiency (\% (95\% CI)) \\
\hline Overall & $48.8(33.3-64.5)$ & $98.5(97.7-99.1)$ & 52.4 & 98.3 & $96.8(95.8-97.8)$ \\
\hline \multicolumn{6}{|l|}{ Age group } \\
\hline $2-5$ & $38.5(13.9-68.4)$ & $99.0(97.2-99.8)$ & 52.4 & 98.3 & $96.6(94.6-98.6)$ \\
\hline $6-8$ & $77.8(40.0-97.2)$ & $98.3(95.2-99.7)$ & 74.1 & 98.6 & $97.3(95.0-99.6)$ \\
\hline $9-11$ & $42.9(9.9-81.6)$ & $100(97.7-100.0)$ & 100 & 99 & $97.6(95.3-99.9)$ \\
\hline $12-14$ & $42.9(17.7-71.1)$ & $97.9(96.4-98.9)$ & 39 & 98.2 & $96.5(95.0-97.9)$ \\
\hline \multicolumn{6}{|l|}{ Sector } \\
\hline Communal & $52.9(27.8-77.0)$ & $98.5(97.2-99.3)$ & 50.9 & 98.6 & $97.3(96.0-98.6)$ \\
\hline Resettlement & $62.5(24.5-91.5)$ & $100.0(98.8-100.0)$ & 100 & 99.3 & $99.0(98.0-100.0)$ \\
\hline LSC & $66.7(22.3-95.7)$ & $99.3(96.2-100.0)$ & 76.9 & 98.9 & $98.0(95.8-100.2)$ \\
\hline SSC & $100(2.5-100.0)$ & $100(94.2-100.0)$ & 100 & 100 & 100 \\
\hline Urban & $18.2(2.3-51.8)$ & $92.5(85.7-96.7)$ & 19.2 & 92 & $85.5(79.1-91.9)$ \\
\hline
\end{tabular}

Mrs M Manyema, Mr W Soko, Mr H Bariri and the research assistants for their assistance in collecting the data and specimens as well as the testing.

\section{References}

1. UNAIDS. Report on the Global HIV/AIDS Epidemic. UNAIDS and WHO; Geneva, Switzerland: UNAIDS/WHO, 2008

. UNAIDS. Report on the Global HIV/AIDS Epidemic. UNAIDS and WHO; Geneva, Switzerland: UNAIDS/WHO, 2003.

3. Granade TC, Phillips SK, Parekh B, Pau CP, George JR. Oral fluid as a specimen for detection and confirmation of antibodies to human immunodeficiency virus type 1. Clinical Diagnostic Laboratory Immunology 1995; 2(4): 395-399.

4. de Almeida OP, de Souza Filho FJ, Scully C, Line SR, Porter S. HIV prevalence in dental outpatients in Brazil. Oral Surg Oral Med Oral Pathol Oral Radiol Endod 1997; 84(4): 365-367.

5. Debattista J, Bryson G, Roudenko N, et al. Pilot of non-invasive (oral fluid) testing for HIV within a clinical setting. Sex Health 2007; 4(2): 105-109.

6. Sherman G, Lilian R, Coovadia A. Oral fluid tests for screening of human immunodeficiency virusexposed infants. Pediatr Infect Dis J 2010; 29(2): 169-172.

7. Sherman G, Jones S. Oral fluid human immunodeficiency virus tests: Improved access to diagnosis for infants in poorly resourced prevention of mother to child transmission programs. Pediatr Infect Dis J 2005; 24(3): 253-256

8. de Moura M, Machedo JE, Kayita J, et al. IgA antibodies to human immunodeficiency virus in serum, saliva and urine for early diagnosis of immunodeficiency virus infection in Ugandan infants. Pediat Infect Dis J 2003; 22(2): 193-195.

9. Woehrle TA, Branson B. New tests for detecting HIV infection. West J Med 1998; 169(6): 371-372.
10. Emmons WW, Paparello SF, Decker CF, Sheffield JM, Lowe-Bey FH. A modified ELISA and Western blot accurately determine anti-human immunodeficiency virus type 1 antibodies in oral fluids obtained with a special collecting device. J Infect Dis 1995; 171(6): 1406-1410.

11. Webber LM, Swanevelder C, Grabow WO, Fourie PB. Evaluation of a rapid test for HIV antibodies in saliva and blood. S Afr Med J 2000; 90(10): 1004-1007.

12. Varghese B, Branson B. Cost and cost-effectiveness of oral fluid HIV testing compared to serum testing. Presented at the 13th International AIDS Conference,. 9 - 14 July 2000, Durban.

13. Pai NP. Rapid oral fluid-based point-of-care HIV testing: applicability in developing countries. Indian Pail NP. Rapid oral fluid-based point-

14. Pai N. Oral fluid-based rapid HIV testing: issues, challenges and research directions. Expert Rev Mol Diagn 2007; 7(4): 325-328.

5. Whatman International. Dried blood spot collection material. www.whatman.com/ DriedBloodSpotCollectionMaterial.aspx (accessed 1 March 2004).

16. Orasure Technologies I. Orasure HIV-1 oral specimen collection device. www.orasure.com/productsinsurance/products-insurance-hiv-specimen.asp. (accessed 1 April 2004).

17. Stark K, Warnecke C, Brinkmann V, Gelderblom HR, Bienzle U, Pauli G. Sensitivity of HIV antibody detection in saliva. Med Microbiol Immunol 1993; 182(3): 147-151

18. Emmons W. Accuracy of oral specimen testing for human immunodeficiency virus. Am J Med 1997; 102(4A): $15-20$.

19. GomoE, Rusakaniko S, Mashange W,MutsvangwaJ, Chandiwana B, MunyatiS. Household Survey of HIV Prevalence and Behaviour in the Chimanimani District, Zimbabwe: A Baseline Survey. Cape Town: HSRC Press, 2006.

20. Hodinka RL, Nagashunmugam T, Malamud D. Detection of human immunodeficiency virus antibodies in oral fluids. Clinical Diagnostic Laboratory Immunology 1998; 5(4): 419-426.

21. Granade TC, Phillips SK, Parekh B, et al. Detection of antibodies to human immunodeficiency virus Granade TC, Phillips SK, Parekh B, et al. Detection of antibodies to human immunodeficiency virus
type 1 in oral fluids: a large-scale evaluation of immunoassay performance. Clinical Diagnostic Laboratory Immunology 1998; 5(2): 171-175.

Accepted 9 August 2010 